\title{
Evaluation of REDCap to Supplement Foodborne Disease Surveillance Systems
}

\section{Mugdha Golwalkar ${ }^{1}$, Kailey Lewis ${ }^{1}$, Marcy McMillian ${ }^{1}$, Heather Mendez ${ }^{1}$, 2 , Katie Garman ${ }^{1}$, John Dunn ${ }^{1}$, Steffany J. Cavallo ${ }^{1}$}

${ }^{1}$ Tennessee Department of Health, Nashville, Tennessee, United States, ${ }^{2}$ Centers for Disease Control and Prevention Public Health Associate Program, Atlanta, Georgia, United States

\section{Objective}

The objective of this study is to evaluate the use of a supplementary data management application to meet surveillance demands for foodborne disease in Tennessee and to highlight successes, challenges, and opportunities identified through this process.

\section{Introduction}

The Tennessee Department of Health (TDH) Foodborne Disease Program conducts routine surveillance for foodborne illnesses and enteric disease outbreaks and participates in statewide enhanced surveillance as part of the Foodborne Disease Center for Outbreak Response Enhancement (FoodCORE) and the Foodborne Diseases Active Surveillance Network (FoodNet) supported by the Centers for Disease Control and Prevention (CDC). TDH uses the CDC NEDSS Base System (NBS) application for routine disease surveillance. However, NBS serves multiple disease programs within TDH and modifications to the system for the rapidly changing data demands, grant requirements, and outbreak needs of the foodborne program, may not be a priority for the system as a whole. In 2014, the TDH Foodborne Disease Program began using the Research Electronic Data Capture (REDCap) application as a solution to changing surveillance needs.

FoodCORE, FoodNet, and routine surveillance data elements are entered into REDCap to supplement NBS, depending on program specific needs and system capability.

\section{Methods}

REDCap was queried for FoodCORE, FoodNet, and routine surveillance projects. Projects were categorized by surveillance activity type. Epidemiologists provided qualitative feedback on successes and challenges in using REDCap to supplement NBS, which were then categorized into attributes according to existing frameworks for evaluating public health surveillance systems $[1,2]$.

\section{Results}

As of August 2018, the TDH Foodborne program housed 45 individual REDCap databases dedicated to surveillance. Four primary database categories were identified: routine case-based surveillance (8), enhanced/active surveillance (6), aggregate outbreak/cluster surveillance tracking (6), and outbreak-specific databases (25). The REDCap application programming interface (API) and an open database connection to NBS within SAS 9.4 (Cary, NC) were used to create unilateral data flow from NBS to REDCap, where possible. Successes and challenges in using REDCap fell into six main surveillance system attributes: Flexibility, Ease of Data Management, Stability, Simplicity, Efficiency, and Acceptability. Successes included the high level of control over data and databases offered by REDCap, the flexibility to rapidly implement program-specific changes, and the accessibility and reliability of REDCap as a de facto back-up of NBS data.

Challenges included lack of interoperability between REDCap databases and with NBS, leading to dual data entry, overuse of REDCap resulting in unnecessarily complex and decentralized data storage (Figure 1), and increased personnel time on data management and extraction for metrics and reports.

\section{Conclusions}

Using REDCap in Tennessee to supplement an existing disease surveillance application increased flexibility and functionality of the foodborne disease surveillance system, but also added complexity and time involved in data management. The Nationally Notifiable Diseases Surveillance System Modernization Initiative (NMI) is developing a standardized message mapping guide (MMG) in collaboration with states and CDC, which incorporates FoodNet data elements and would transition data collection tools

SDS Annual Conference Proceedings 2019. This is an Open Access article distributed under the terms of the Creative Commons AttributionNoncommercial 4.0 Unported License (http://creativecommons.org/licenses/by-nc/3.0/), permitting all non-commercial use, distribution, and reproduction in any medium, provided the original work is properly cited. 
in NBS for foodborne diseases to a more portable and flexible format. Implementation of this MMG could minimize case-based data entry into REDCap. Tools that offer increased interoperability between NBS and REDCap and between REDCap databases could also improve the efficiency of using complementary applications for rapidly changing foodborne disease surveillance needs.

\section{Acknowledgement}

Authors would like to acknowledge Corinne Davis, REDCap administrator, for her assistance in creating and organizing databases for the Foodborne Diseases Program.

\section{References}

1. German RR, Lee LM, Horan JM, Milstein RL, Pertowski CA, Waller MN. Updated guidelines for evaluating public health surveillance systems: recommendations from the guidelines working group. MMWR Recomm Rep. 2001;50(Rr-13):1-35.

2. Calba C, Goutard FL, Hoinville L, et al. 2015. Surveillance systems evaluation: a systematic review of the existing approaches. BMC Public Health. 15, 448. doi:https://doi.org/10.1186/s12889-015-1791-5. PubMed

Figure 1: Data and surveillance process flow for the Tennessee Department of Health Foodborne Program. Each REDCap database box reflects a category containing the number of databases specified in parentheses.

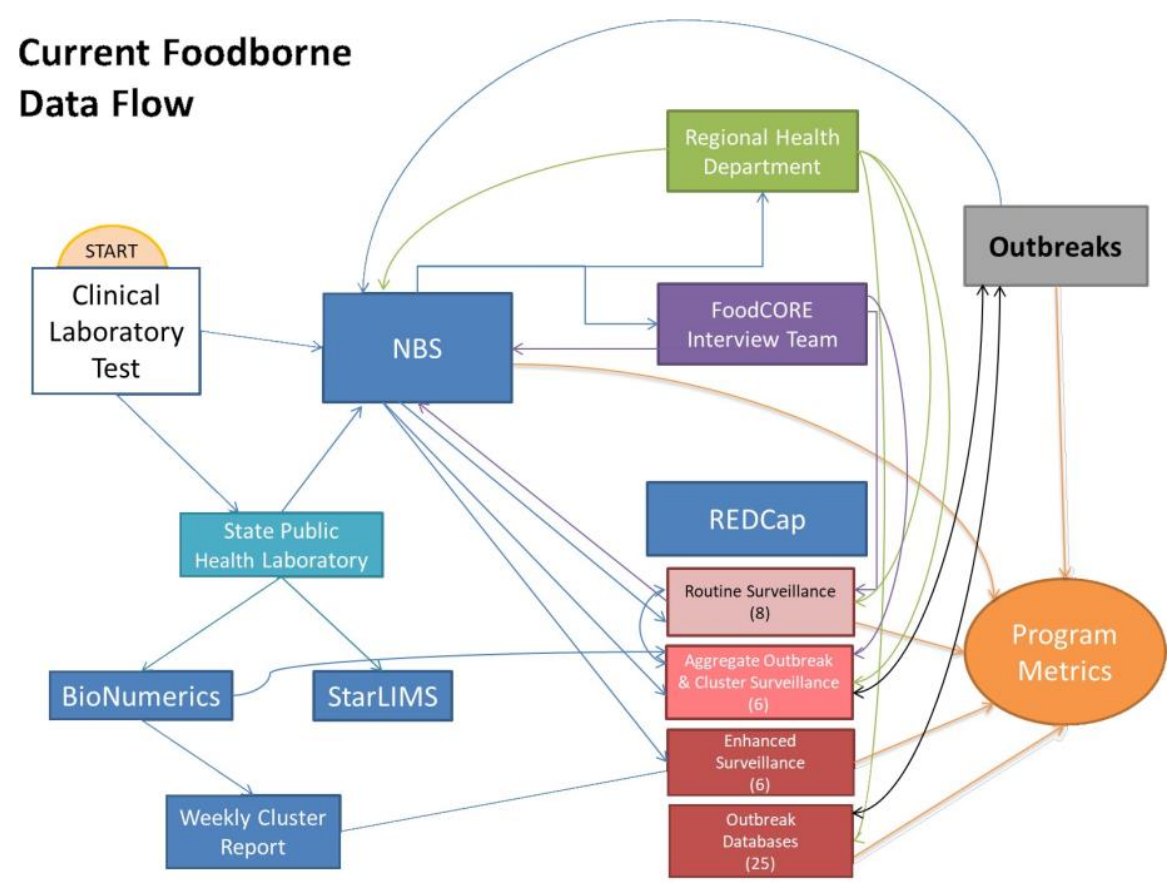

ISDS Annual Conference Proceedings 2019. This is an Open Access article distributed under the terms of the Creative Commons AttributionNoncommercial 4.0 Unported License (http://creativecommons.org/licenses/by-nc/3.0/), permitting all non-commercial use, distribution, and reproduction in any medium, provided the original work is properly cited. 